\title{
Rolling Bearing Diagnosis Based on the Higher Order Spectra
}

\author{
Len Gelman ${ }^{1}$, Tejas H. Patel ${ }^{2}$, and Brian Murray ${ }^{3}$ Allan Thomson ${ }^{4}$ \\ ${ }^{1,2}$ Cranfield University, UK. \\ L.Gelman@cranfield.ac.uk \\ t.patel@cranfield.ac.uk \\ ${ }^{3,4} S K F, U K$ \\ Brian.Murray@skf.com \\ Allan.Thomson@skf.com
}

\begin{abstract}
Bearing defect diagnosis is traditionally done using the demodulation/enveloping technology. Diagnosis is mostly based on the spectrum of the squared envelope signal. In literature, the use of the higher order spectra (HOS) has shown to have a tremendous potential for vibration based diagnostics. In this paper we implemented and experimentally validated the higher order spectra based on the envelope analysis for the diagnosis of ball bearing defects. The implemented technology employs the spectral kurtosis to obtain a frequency band for the demodulation and the third order normalized spectra, i.e. the bicoherence for diagnosis of bearing fault. The high effectiveness of the diagnostics of the implemented technology has been experimentally revealed and compared with that of wellknown demodulation/enveloping technology.
\end{abstract}

\section{INTRODUCTION}

Diagnostics and prognostics of the rolling element bearing has been widely researched, e.g. works of McFadden and Smith (1984), Tandon and Choudhury (1999), Randall and Antoni (2011), Wang, Youn, and $\mathrm{Hu}$ (2012), Camci, Medjaher, Zerhouni, and Nectoux (2012), Hu, Youn, and Wang (2012), and Medjaher, Camci, and Zerhouni (2012). The demodulation/enveloping based methods have a long history of successful application for the bearing damage diagnosis (McFadden \& Smith, 1984; Tandon \& Choudhury, 1999; Randall \& Antoni, 2011). Every time defect passes through a rolling element an impact occurs and an impulse response is generated. This short duration impact causes resonance of associated rolling element or even of the machine, which manifests through modulation by bearing characteristic defect frequency. The main purpose

L. Gelman et al. This is an open-access article distributed under the terms of the Creative Commons Attribution 3.0 United States License, which permits unrestricted use, distribution, and reproduction in any medium, provided the original author and source are credited. of demodulation/enveloping technique is to find the modulating (carrier) frequency, which is believed to be related to excited resonance frequency. Then the signal is band-pass filtered around the modulating (carrier) frequency and shifted to the DC. The main challenge in application of this method lies in efficiently detecting or filtering out frequency band/range for demodulation. The one of the approach was to find resonance from its broadband present in high frequency region of the spectrum (McFadden \& Smith, 1984), however, this need historical data of healthy bearing for comparison purpose (Sawalhi \& Randall, 2004). Furthermore, presence of high amplitude interference, e.g. shaft rotation frequency and its harmonics, gear mesh frequencies, etc. may make detection of low amplitude resonance difficult. Enveloping based on wavelets has shown to have excellent adaptability for successful application for bearing fault diagnosis (Nikolaou \& Antoniadis, 2002). The spectral kurtosis (SK) has become widely applied tool for selection of demodulation band since last decade (Randall \& Antoni, 2011; Sawalhi \& Randall, 2004; Antoni, 2006; Wang \& Liang, 2011). Compared to wavelets, the SK is computationally fast and could easily be incorporated into portable instrument for on-field applications. The final diagnosis is based on the spectrum of the squared envelope. Appearance of particular bearing characteristic defect frequency and its harmonics in the spectrum would tell the type of bearing defect, i.e. cage defect, race defect, or rolling element (ball or roller) defect.

In this study we performed higher order spectral analysis of the squared envelope obtained using the SK based optimal de-noising (Wiener) filter. The use of higher order spectra (HOS) is widely investigated for damage diagnosis and classification (Howard, 1997; Jiang, Liu, Li, \& Tang, 2011; Gelman, White, \& Hammond, 2005). The use of bicoherence for bearing condition monitoring has been demonstrated by $\mathrm{Li}, \mathrm{Ma}$, and Hwang (1996) and Yang, Stronach, and MacConnell (2002) using the raw vibration signal. They inputted bicoherence features in linear 
discriminant classifier (Li et al, 1996) and artificial neural networks (Yang et al, 2002) respectively. The present work differs from the past works in the following three ways:

(1) The amplitude envelope signal is used for HOS calculation

(2) The HOS calculation performed around bearing characteristic frequency only. Thus, the implemented method is computationally fast

(3) A comparison is made with the classical demodulation/enveloping method based on the envelope spectrum. A diagnostic feature from squared envelope spectra is obtained and compared with the normalized higher order spectral feature using a statistical effectiveness measure, the Fisher criterion (Webb, 2003).

\section{EXPERIMENTAL SETUP}

The bearing test rig has a coupled VSD (variable speed drive) motor driving a shaft supported on three identical bearings (FK UCP203). Table 1 lists the test bearing geometric dimensions and the defect frequencies. Figure 1 shows the test rig under consideration. The VSD drive provides 20-60 Hz supply frequency. The test rig has provision for inducing load at the non-drive end (NDE) through misalignment.

\begin{tabular}{cc}
\hline Number of balls & 8 \\
\hline Bore diameter & $17 \mathrm{~mm}$ \\
\hline Outside diameter & $40 \mathrm{~mm}$ \\
\hline Ball diameter & $6.75 \mathrm{~mm}$ \\
\hline $\begin{array}{c}\text { Ball pass frequency, } \\
\text { inner race, } B P F I\end{array}$ & $4.95 * f_{r}$ \\
\hline $\begin{array}{c}\text { Ball pass frequency, } \\
\text { outer race, BPFO }\end{array}$ & $3.05 * f_{r}$ \\
\hline $\begin{array}{c}\text { Ball spin frequency, } B S F \\
\text { Fundamental train frequency, } \\
F T F\end{array}$ & $0.382 * f_{r}$ \\
\hline
\end{tabular}

Table 1. Test bearing specifications and frequencies

Tests were conducted at full speed and full load condition. The full speed and full load condition corresponds to $60 \mathrm{~Hz}$ supply frequency and $196 \mathrm{~N}$ resultant radial load. The misalignment radial load was induced though using premachined shims at the non-drive end (NDE). The measurement of the resultant radial load was done in a separate test. The experiment starts with all rotational components at rest. The motor was then made to rotate at set rotational speed of through the VSD. Experiments were conducted first with the healthy bearing and then with the defective bearings. The test bearing was placed on the nondrive end (NDE). The drive end (DE) and middle bearings were never changed during experiments. Bearings with inner race defect, outer race defect and cage defect were tested in the sequence at the same operating speed and load. Although the VSD set rotational frequency was $60 \mathrm{~Hz}$, the actual speed of rotation was found to be $59 \mathrm{~Hz}$. Figure 2 show pictures of the defective bearings. The defect sizes are show in the Table 2.

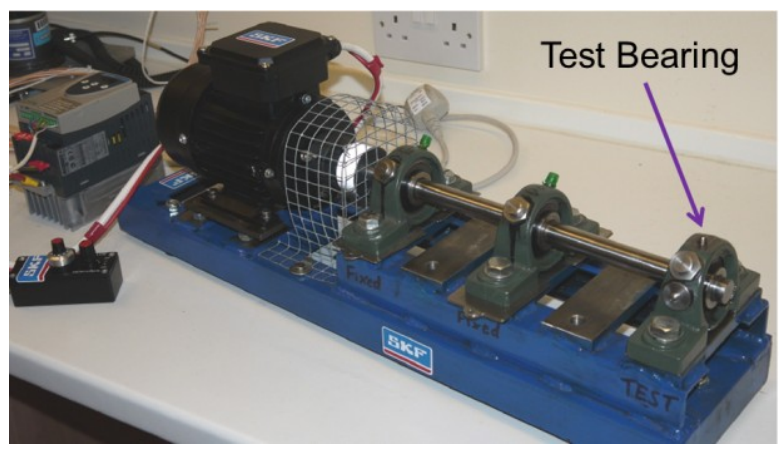

Figure 1. The bearing test-rig

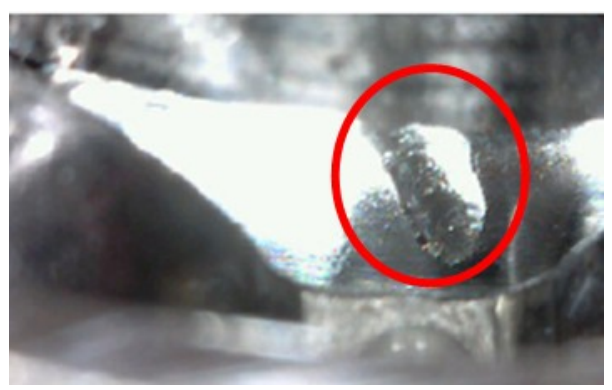

(a) Inner race defect

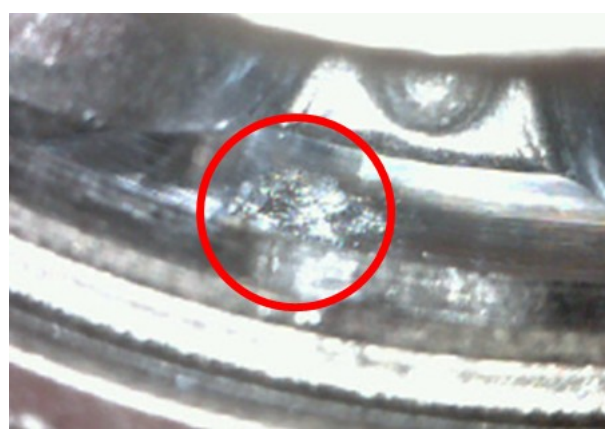

(b) Outer race defect

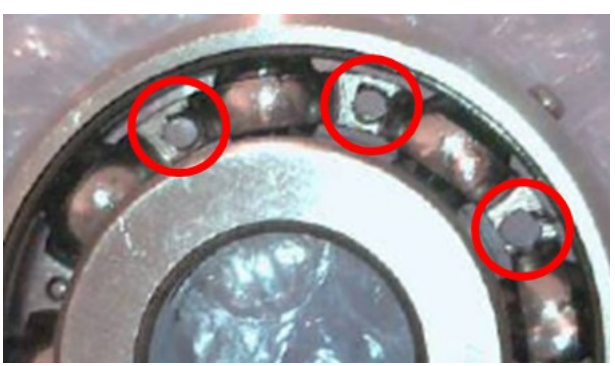

(c) Cage defect

Figure 2. Bearing defects 
Vibration signal was captured from a unidirectional accelerometer (amplitude response: $0.5-5 \mathrm{kHz}( \pm 5 \%)$; sensitivity: $5.21 \mathrm{pC} / \mathrm{ms}^{2}$; resonance frequency: $25 \mathrm{kHz}$ ) placed along vertical direction on the test bearing housing. Active antialiasing filter (cut-off frequency $=13.5 \mathrm{kHz}$ ) was used before capturing the digitized data. A speed reference signal (1 pulse per shaft revolution) was also captured synchronously. The data acquisition was done at $40 \mathrm{kHz}$ in order to provide necessary bandwidth for channel recording.

\begin{tabular}{ll}
\hline Inner race & $0.8 \mathrm{~mm}$ circumferential length and \\
defect & $0.2 \mathrm{~mm}$ deep \\
\hline Outer race & $1.6 \mathrm{~mm}$ circumferential length and \\
defect & $0.2 \mathrm{~mm}$ deep \\
\hline Cage defect & 3 sequential rivets removed \\
\hline
\end{tabular}

Table 2. Bearing defect size

\section{HOS ANALYSIS BASED ON ENVELOPE}

\subsection{The Diagnostic Feature from HOS of Defect Frequency}

The HOS of order 3 (i.e. $n=3$ ), known as bispectrum, is well-known to ascertain the nonlinear phase coupling between fundamental defect frequency and their harmonics (Gelman, Lapena, \& Thompson, 2009). In practice, the normalized bispectrum, i.e. bicoherence is used (Collis, White, \& Hammond, 1998). The generalized bicoherence can be written as,

$$
b\left(f_{i}, f_{j}\right)=\frac{\sum_{m=1}^{M} X_{m}\left(f_{i}\right) X_{m}\left(f_{j}\right) X_{m}^{*}\left(f_{i}+f_{j}\right)}{\sqrt{\sum_{m=1}^{M}\left|X_{m}\left(f_{i}\right) X_{m}\left(f_{j}\right)\right|^{2}} \sqrt{\sum_{m=1}^{M}\left|X_{m}\left(f_{i}+f_{j}\right)\right|^{2}}}
$$

Where $X_{m}\left(f_{i}\right)$ and $X_{m}\left(f_{j}\right)$ are the Discrete Fourier Transforms (DFTs) of the $m^{\text {th }}$ segment of the signal $x_{m}(t)$ at frequency $f_{i}$ and $f_{j}$ respectively, $M$ is the total number of segments, * indicates complex conjugate. Presence of defect is revealed through strong coupling between fundamental defect frequency and its harmonics, resulting in the bicoherence value, i.e. $\left|b\left(f_{i}, f_{j}\right)\right|$, almost equal to 1 . The no-defect on the other hand has almost no coupling and revealed through a low value (almost equal to 0) of bicoherence. In this study, frequency up to $3^{\text {rd }}$ harmonic of the bearing defect frequency is considered. i.e. $f_{i}, f_{j}=f, 2 f, 3 f$. where, $f$ is the bearing defect frequency. As evident from Eq. (1) presence of at least second harmonic (i.e. 2f) of the fundamental defect frequency is necessary for bicoherence technology to work. In most practical cases, i.e. nonlinear systems, this is not the problem. However, the cases where higher harmonics are not evident, the bicoherence technology will not work.

\subsection{Feature from Squared Envelope Spectrum}

The diagnostic feature in the form of bicoherence of the bearing defect frequency is calculated using Eq. (1). For comparison purpose, a defect frequency feature is obtained from the squared envelope spectrum. The defect frequency feature is the ratio of summation of spectral amplitude at bearing defect frequency and its harmonics to the surrounding background vibration minus one.

$$
E\left(f_{i}\right)=\frac{\sum_{i=1}^{N_{h}} X_{m}\left(f_{i}\right)}{\sum_{i=1}^{N_{h}} X_{n}\left(f_{i}\right)}-1
$$

Where, $X_{m}\left(f_{i}\right)$ is the spectral amplitude at frequency $f_{i} . X_{n}\left(f_{i}\right)$ is the mean background vibration around $f_{i} . f_{i}=f, 2 f, 3 f$. where, $f$ is the characteristic frequency of bearing defect and its harmonics. $N_{h}$ is the number of harmonics. As mentioned in Section 3.1, $N_{h}=3$ is considered. The estimation of $X_{n}$ is done within $\left(f_{i}-0.25 * f_{\text {shaft }}\right]$ and $\left(f_{i}+0.25 * f_{\text {shaft }}\right]$. Figure 3 is a sample spectrum of the squared envelope graphically showing $X_{m}$ and $X_{n}$ at defect frequency, $f_{i}=f$, for inner race defect at full speed shaft rotation, $f_{\text {shaft }}=57.95 \mathrm{~Hz}$. The calculation details for the same are provided in Section 3.4.

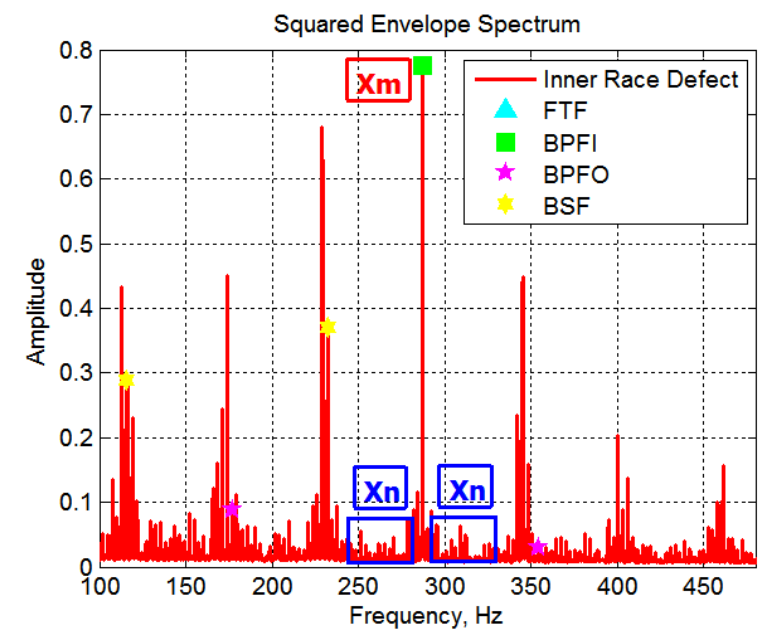

Figure 3. Squared Envelope Spectrum

\subsection{Procedure for Bicoherence Feature Calculation}

Following steps should be undertaken to calculate bicoherence feature of Eq. (1). Figure 4 shows the flow chart of the same.

Step 1: Perform angular resampling of the raw vibration signal to compensate for slight fluctuation of the shaft rotation speed.

Step 2: Divide the angular resampled signal $x(t)$ into overlapping blocks $x_{v}(t)$ by the external window $h_{e}(t) ; v=1$, $\ldots, V, V$ defines the total number of blocks $x_{v}(t), T$ is the time center of the external window. The external window $h_{e}(t)$ could be of any type, i.e. rectangular, Hamming, Chebyshev, etc. The overlap of the external window should 
be adapted to adjust the variations in the signal. For example, signal with fast variation in speed need higher level of overlapping along with the shorter window size. The signal under consideration was collected at steady speed and load condition, therefore, overlap of the external window is not a critical parameter. Since the acquired single is of limited time length, we used high level of external overlapping in order to increase feature value statistics. In this study we used rectangular external window of length $100 \mathrm{sec}$ and $30 \mathrm{sec}$, and overlap of $99 \%$ and $90 \%$ respectively for bicoherence and defect frequency feature calculations.

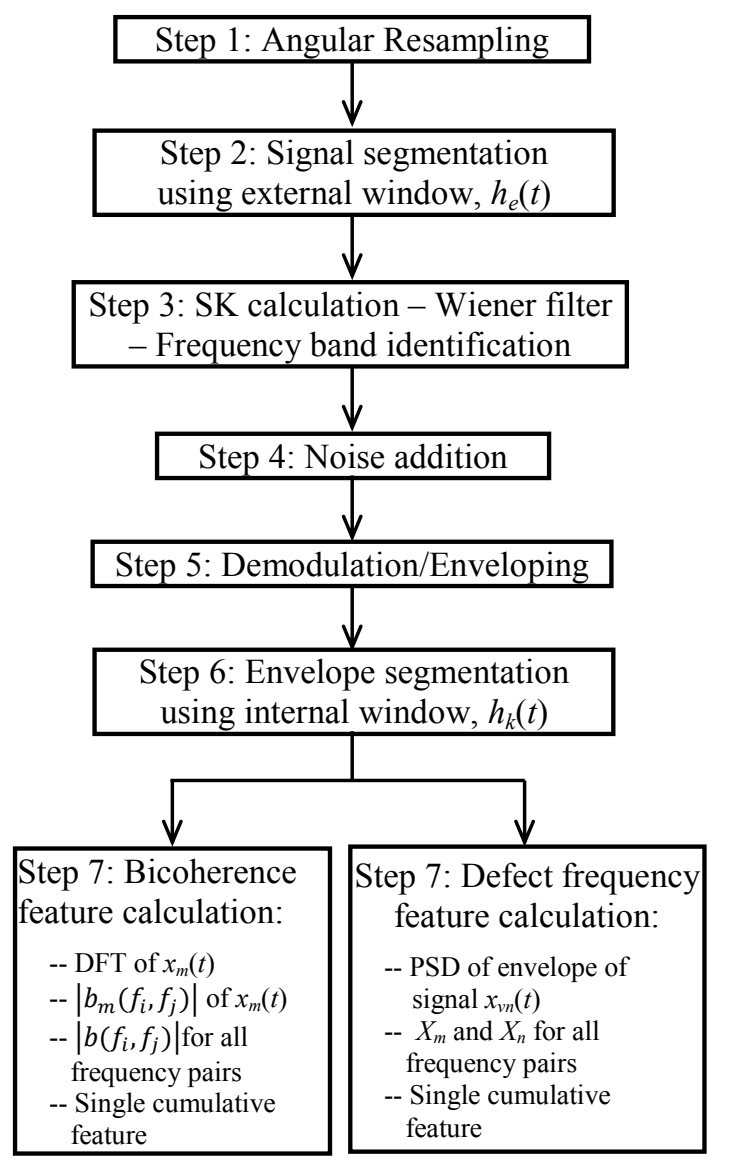

Figure 4. Procedure for Feature Calculation

Step 3: Identify resonant frequency bands using the spectral kurtosis (SK) based optimal denoising (Wiener) filter from $x_{v}(t)$. The procedure for obtaining SK based de-noising filter is widely report (Randall \& Antoni, 2011; Sawalhi \& Randall, 2004; Antoni, 2006), therefor, related discussion omitted here. For the test-rig and experiment conditions under consideration, it was observed that the impulse response duration due to defect impact is shorter than the time between impacts, i.e. damping of the test-rig is high. To find the most suitable SK parameters, we have used a procedure similar to the kurtogram. In this we observe the kurtosis value over several realizations of the signal, i.e. $x_{v}(t)$ at different window sizes and thresholds. A window length of $1 / 10$ times the duration between impacts, which corresponds to a frequency resolution of 10 times the bearing characteristic defect frequency, found the most suitable for the spectral kurtosis calculation. The significance threshold of $1 \%$ was used to obtain the Wiener filter from the spectral kurtosis. Comparison of sample spectral kurtosis plot in Figure 5 clearly shows the frequency bands (around 5, 10 and $15 \mathrm{kHz}$ ) excited by the inner race defect.

For bearings with high speed and low damping, the impact duration is longer than the time between impacts, thus causing the so called "smearing effect", which can affect the quality of the proposed feature. As mentioned by reviewers and showed by Randall and Antoni (2011), the Minimum Entropy Deconvolution (MED) can reduce the smearing effect. The MED should be included just before the SK calculation in the procedure shown in Figure 4.

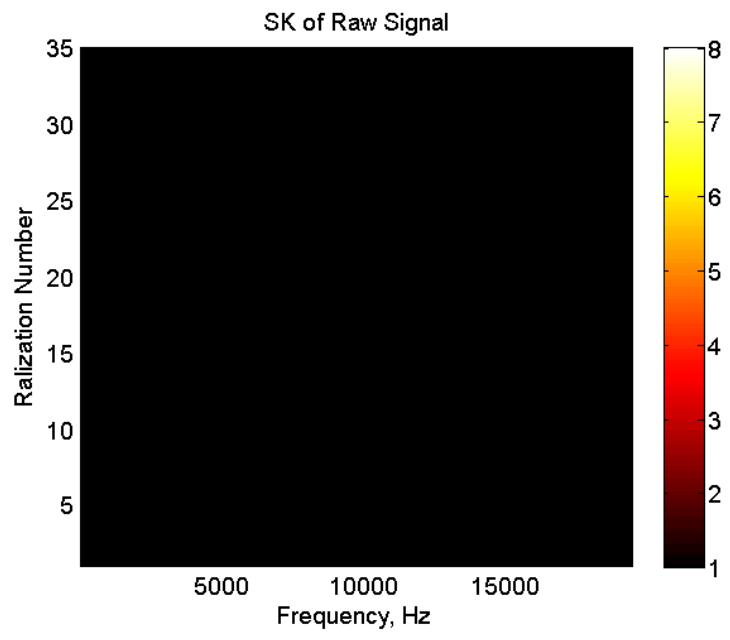

(a) No damage

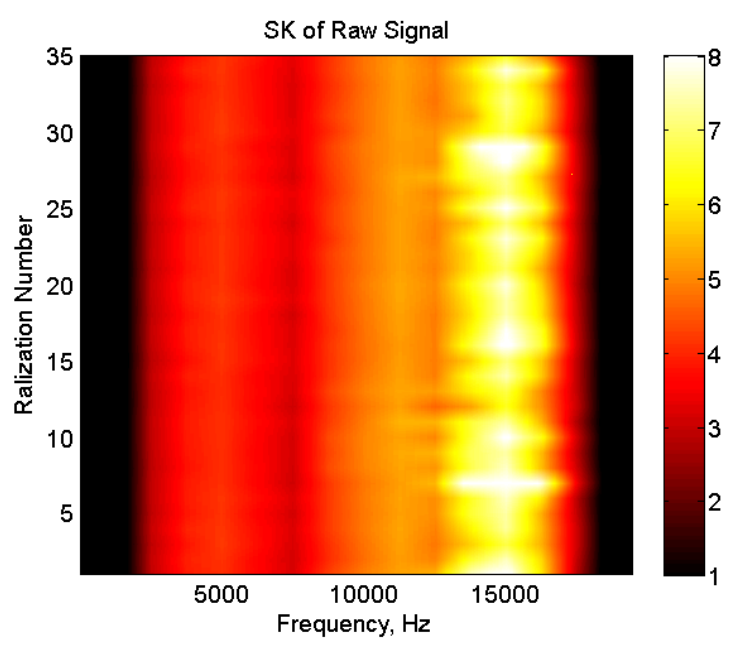

(b) Inner race defect

Figure 5. Spectral kurtosis over few realizations 
Step 4: Add white Gaussian noise to the signal $x_{v}(t)$ to obtain $x_{v n}(t)$. Although, the noise addition can never substitute the complexity and the nonlinearity observed in on-field applications, to some extent it relates to them. First the signal power was measured and then white Gaussian noise of prescribed signal to noise ratio (SNR) was added. The SNRs of $10 \mathrm{~dB}, 5 \mathrm{~dB}$, and $-5 \mathrm{~dB}$ are considered. It is important to note that this step is optional not the requirement of the technology.

Step 5: Obtain envelope (i.e. squared) signal after demodulating signal $x_{v n}(t)$ in frequency band identified in step \#3 above.

Step 6: Divide the squared envelope signal into overlapping segments $x_{m}(t)$ by the internal window $h_{k}(t), m=1, \ldots, M, M$ defines the total number of segments $x_{m}(t)$ in the time block. The internal window $h_{k}(t)$ and internal overlap could be different from the external window $h_{e}(t)$ and external overlap. It is important to note that the requirement on the frequency resolution governs the internal window length. The Hamming internal window of $5 \mathrm{sec}$ (frequency resolution $=0.2 \mathrm{~Hz}$ ) with overlap of $66 \%$ is used in this study. Figure 6 graphically illustrates the internal windows inside a rectangular external window.

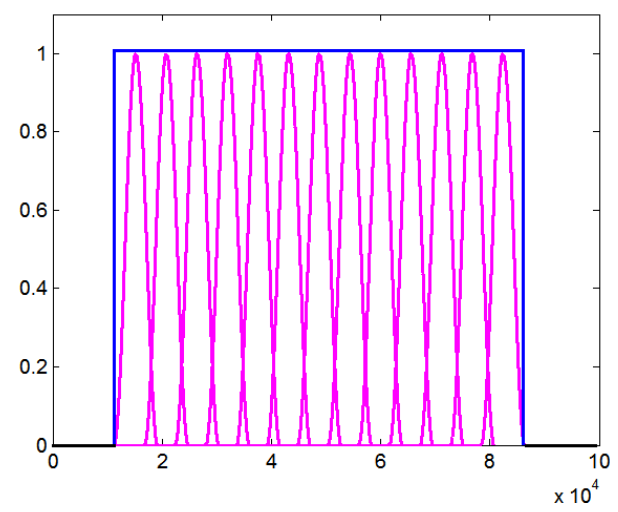

Figure 6. Illustration of internal and external windows

Step 7: Calculate bicoherence feature (Eq. 1) using the following steps.

- Compute DFT of segment $x_{m}(t)$ and obtain $X_{m}(f)$.

- Compute numerator and denominator around $f_{i} \pm \Delta f_{i}$ and $f_{j} \pm \Delta f_{j}$. It may be noted that $\Delta f$ accounts for slight smearing of defect frequency due to random slippage of rolling element between races. The $\Delta f$ is considered to be $0.6 \%$ of the frequency concerned. The $\left|b_{m}\left(f_{i}, f_{j}\right)\right|$ is the ratio of maximum of numerator and maximum of denominator obtained within $f_{i} \pm \Delta f_{i}$ and $f_{j} \pm \Delta f_{j}$.

- Repeat above steps for $m=1, \ldots, M$ and obtain the statistical estimate of the bicoherence by averaging over $M$ segments.
- Repeat above steps for all frequency pairs of interest, i.e. $f_{i}, f_{j}=f, 2 f, 3 f$. The diagnostic feature is the cumulative sum of $\left|b\left(f_{i}, f_{j}\right)\right|$ for all frequency pairs.

\subsection{Procedure for Defect Frequency Feature Calculation}

The procedure to calculate defect frequency feature of Eq. (2) is quite similar to the bicoherence feature calculation. In the following we will only show steps different from bicoherence feature calculation either in calculation or parameters.

Step 2: The rectangular external window of $30 \mathrm{sec}$ with overlap of $90 \%$ is used.

Step 6: The Hamming internal window of $5 \mathrm{sec}$ (frequency resolution $=0.2 \mathrm{~Hz}$ ) with overlap of $50 \%$ is used.

Step 7: Calculate the defect frequency feature (Eq. 2) using the following steps.

- Obtain the averaged power spectral density (PSD) of the squared envelope signal for time block $x_{v n}(t)$ using internal windowing and overlapping parameters mentioned in step \#6.

- Obtain $X_{m}$ through maximum amplitude search around defect frequency $f_{i} \pm \Delta f_{i}$. The $\Delta f_{i}$ is $0.6 \%$ of the frequency concerned, i.e. $f_{i}$.

- Compute background vibration level $X_{n}$ around defect frequency. The estimation of $X_{n}$ is done within $\left(f_{i}-\right.$ $\left.0.25 * f_{\text {shaft }}\right]$ and $\left(f_{i}+0.25 * f_{\text {shaft }}\right]$. The $f_{i}$ is the frequency at which maximum $X_{m}$ was found.

- Repeat above two steps for $N_{h}$ harmonics and compute the defect frequency feature using Eq. (2). $N_{h}=3$ is used in this study.

It is possible to identify more than one resonant frequency band in step \#3 for defective bearing. The steps $\# 5$ to $\# 7$ are repeated for each frequency band and the bicoherence and the defect frequency will be a cumulative sum of the feature value obtained for each band. In cases where the spectral kurtosis does not find a frequency band, e.g. healthy bearing, a feature value of zero is assigned.

\section{APPLICATION OF HOS TEChNOLOGY FOR BEARING DEFECT DiAgNosis}

Figures 7 and 8 compare enveloping technology and $3^{\text {rd }}$ order HOS (i.e. bicoherence) technology through defect frequency and bicoherence features for three different signal to noise ratios, i.e. $\mathrm{SNR}=10 \mathrm{~dB}, 5 \mathrm{~dB}$, and $-5 \mathrm{~dB}$. It can be seen that with increase in noise level the value of defect frequency feature and its variance decreases. The decrease in variance results into a clearer separation between healthy and defective condition. The fluctuation of the defect frequency feature for damage case is due to presence of unknown nonlinearity and/or nonstationarity. 


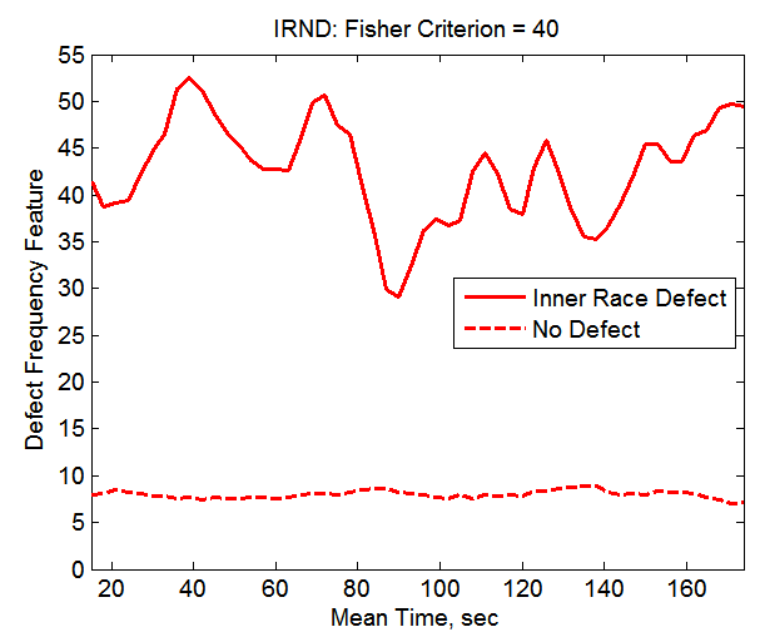

(a)

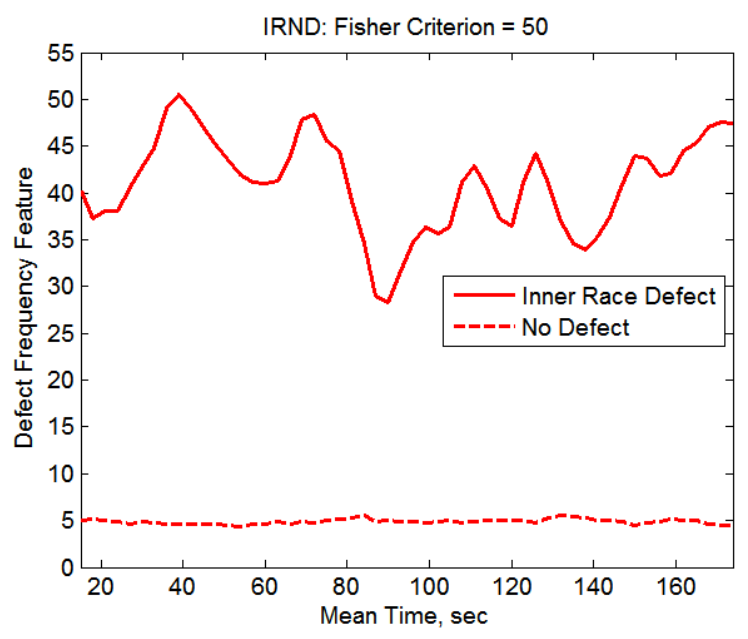

(b)

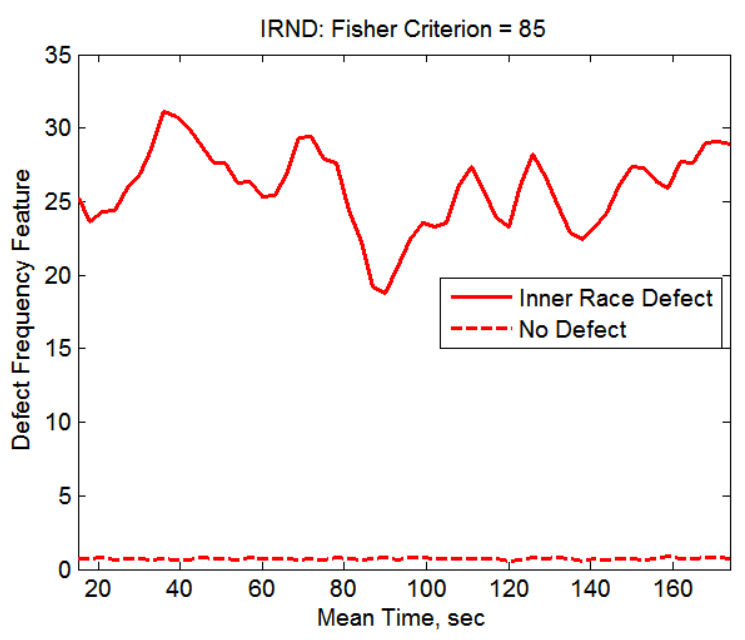

(c)

Figure 7. Defect frequency for inner race defect; (a) SNR = $10 \mathrm{~dB}$, (b) $\mathrm{SNR}=5 \mathrm{~dB}$, (c) $\mathrm{SNR}=-5 \mathrm{~dB}$

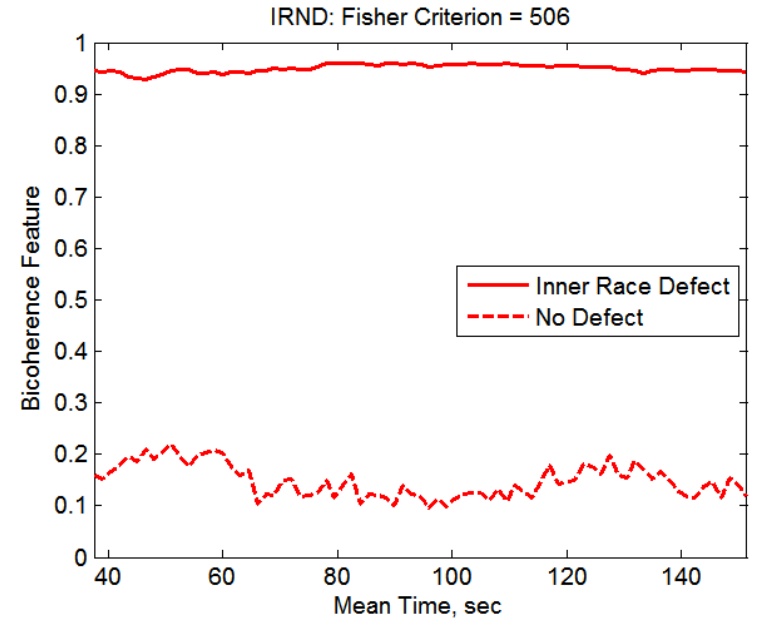

(a)

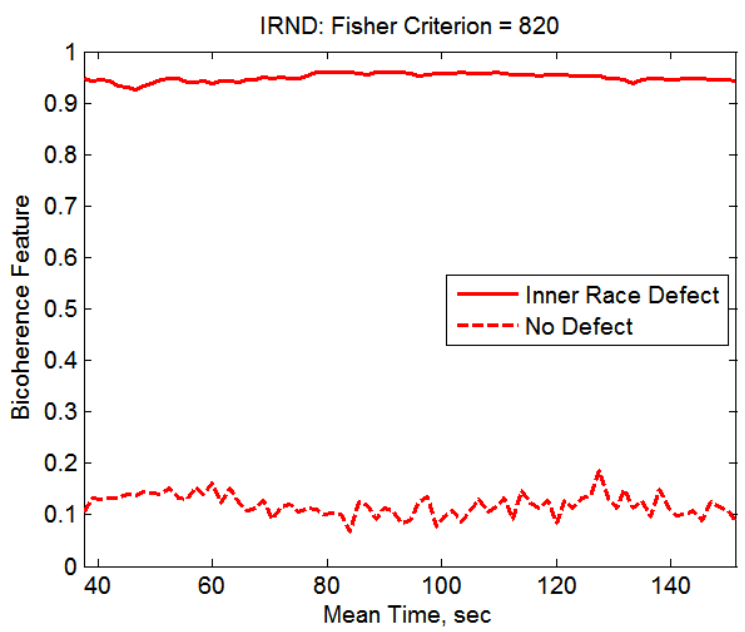

(b)

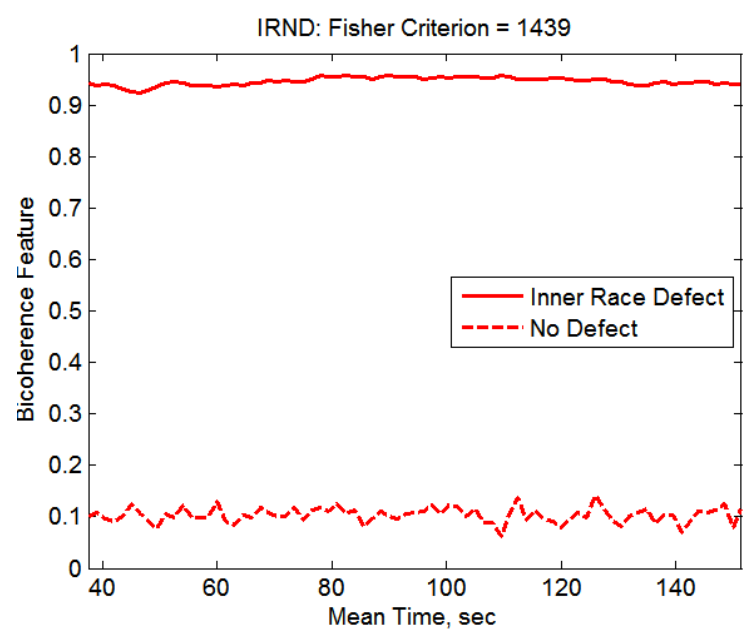

(c)

Figure 8. Bicoherence feature for inner race defect; (a) SNR $=10 \mathrm{~dB}$, (b) $\mathrm{SNR}=5 \mathrm{~dB}$, (c) $\mathrm{SNR}=-5 \mathrm{~dB}$ 
For bicoherence, the feature value more or less remains the same at different noise levels for the defective bearing. This endorses the fact of insensitiveness of bicoherence to the noise. The higher level of noise can suppress the low level of coupling between defect frequency and their harmonics that could possibly exists for healthy bearing. This is manifested by decrease in bicoherence feature with increase in noise level for the healthy bearing.

As can be seen from Figures 7 and 8, both the methods (i.e. defect frequency feature and bicoherence feature) show a clear separation between no-defect and defect case. A simple threshold would tell the faulty states (i.e. no-defect and defect) apart for both the methods. So, it is necessary to quantify the separation between two faulty states. This has been done by using a statistical measure in the form of Fisher criterion. The Fisher criterion (Eq. 3) is defined (Webb, 2003) as the square of the mean difference divided by the variance sum.

$F C(\theta)=\frac{\left(\mu_{D}-\mu_{N D}\right)^{2}}{\sigma_{D}^{2}+\sigma_{N D}^{2}}$

where $\mu$ and $\sigma$ are respectively the mean and standard deviation of the features, suffixes $D$ and $N D$ refer to the damaged and undamaged conditions.

The defect frequency feature (Eq. 2) has high value of mean difference (Figure 7), whereas, the bicoherence feature (Figure 8) has low value of variance. Since the Fisher criterion captures both these aspects, it is best suited for the study presented here.

The Fisher criterion (shown above each figure) increases with increase in noise level for both defect frequency and bicoherence feature. The Fisher criterion for bicoherence is always high, i.e. 12 to 17 times, compared to that of defect frequency feature.

Figures 9 and 10 show the defect frequency and bicoherence feature for outer race and cage defect for the same speed and load condition of the test. Instead of comparing features at different noise levels, the noise level at which the best separation between healthy and defective condition achieved for both bicoherence and enveloping technologies are shown. The signal to noise ratios (SNRs) of $5 \mathrm{~dB}$ and $5 \mathrm{~dB}$ were found best for defect frequency feature and bicoherence feature respectively for Figures 9 and 10.

Similar to the inner race defect, the separation between faulty cases is present for other SNR values too but with slightly lower Fisher criterion value. For outer race and cage defects too, the Fisher criterion for bicoherence technology is always higher compared to the enveloping technology. Table 3 presents a ready comparison of Fisher criterion for both the methods.

\begin{tabular}{lccc}
\hline Defect & \multicolumn{3}{c}{ Fisher criterion } \\
& $\begin{array}{c}\text { Bicoherence } \\
\text { feature, } F C_{b c}\end{array}$ & $\begin{array}{c}\text { Defect frequency } \\
\text { feature, } F C_{d f}\end{array}$ & $\begin{array}{c}\text { Gain } \\
F C_{b c} / F C_{d f}\end{array}$ \\
\hline Inner race & 1439 & 85 & 16.9 \\
\hline Outer race & 282 & 164 & 1.7 \\
\hline Cage & 817 & 23 & 35.5 \\
\hline
\end{tabular}

Table 3. Fisher criterion values

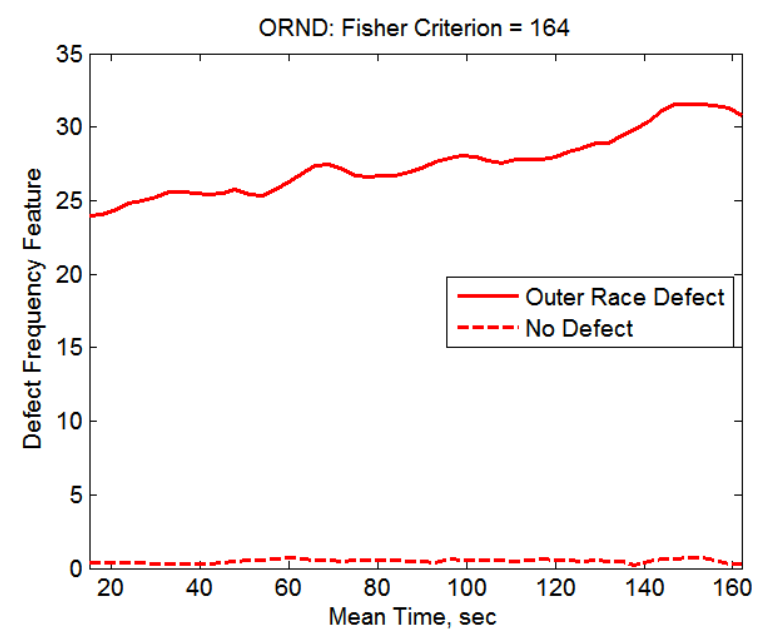

(a)

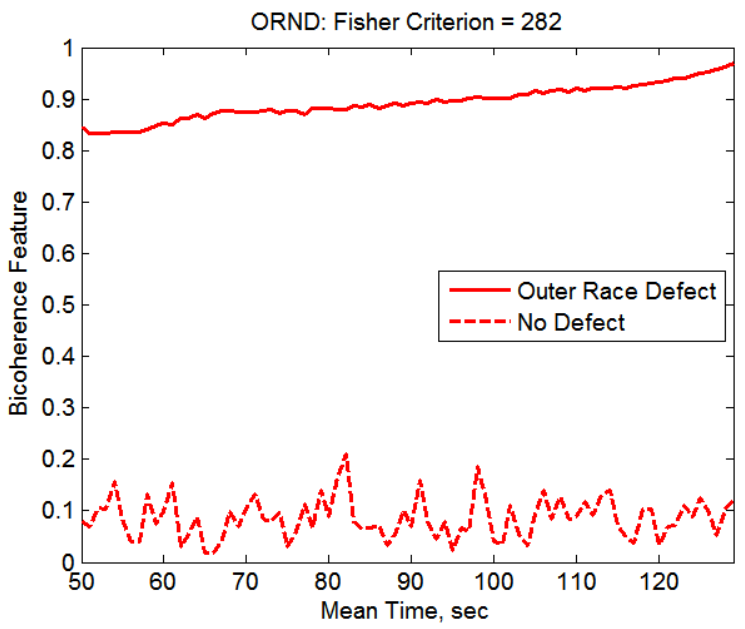

(b)

Figure 9. Outer race defect; (a) defect frequency feature, $\mathrm{SNR}=5 \mathrm{~dB}$; (b) bicoherence feature, $\mathrm{SNR}=-5 \mathrm{~dB}$

\section{Conclusions}

The bicoherence technology based on the envelope analysis is implemented and experimentally validated in this paper. The proposed technology employs the spectral kurtosis for identification of frequency bands for demodulation/envelope extraction. 
The technology is successfully validated on experimental laboratory vibration data related to undamaged bearings and low levels of the following bearing faults:

- $\quad$ outer race local damage

- inner race local damage

- ball and cage local damages

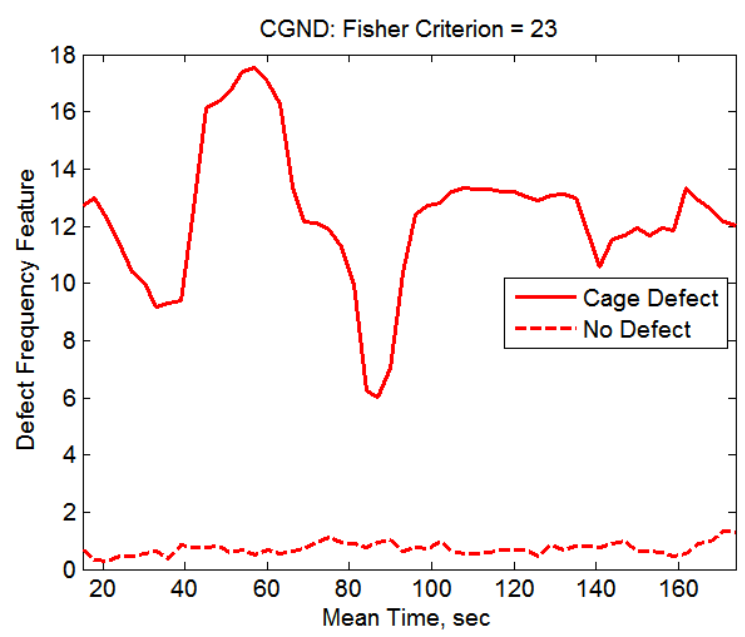

(a)

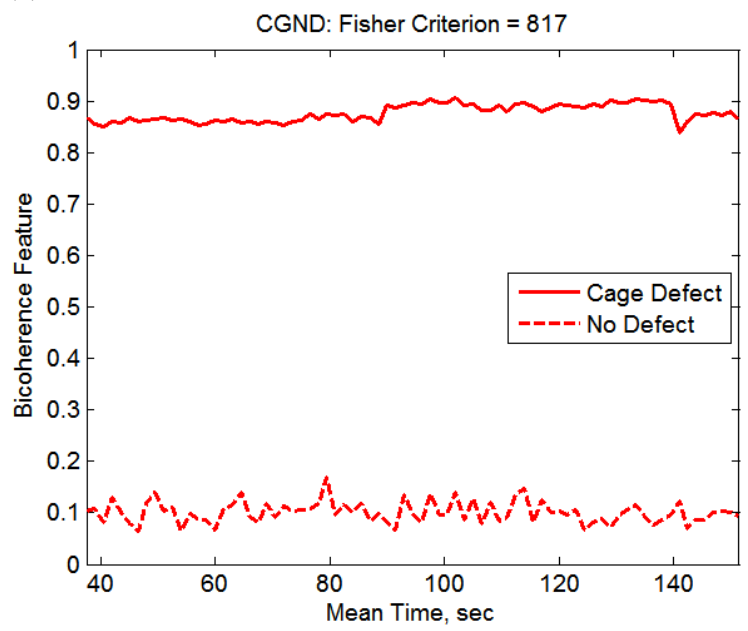

(b)

Figure 10. Cage defect; (a) defect frequency feature, $\mathrm{SNR}=5 \mathrm{~dB}$; (b) bicoherence feature, $\mathrm{SNR}=-5 \mathrm{~dB}$

A comparison is made between the implemented technology and the classical demodulation technology in terms of the Fisher criterion of the diagnostic feature. By using laboratory experimental data for three kinds of bearing defects, it has been shown that the implemented technology offers a gain of 1.7, 16.9 and 35.5 times in terms of the Fisher criterion for outer race, inner race and cage/ball defects respectively.
Since the implemented technology involves HOS calculation just around the bearing defect frequencies, the technology is computationally fast and could easily be incorporated into portable condition monitoring instruments for on-field applications.

\section{REFERENCES}

McFadden, P., and Smith, J. (1984). Vibration monitoring of rolling element bearings by the high frequency resonance technique - a review. Tribology International, vol. 17, pp. 3-10. doi:10.1016/0301679X(84)90076-8

Tandon, N., and Choudhury, A. (1999). A review of vibration and acoustic measurement methods for the detection of defects in rolling element bearings. Tribology International, vol. 33, pp. 469-480. doi: 10.1016/S0301-679X(99)00077-8

Randall, R., and Antoni, J. (2011). Rolling element bearing diagnostics - A tutorial. Mechanical Systems and Signal Processing, vol. 25, pp. 485-520. doi: 10.1016/j.ymssp.2010.07.017

Wang, P., Youn, B.D., and Hu, C. (2012). A generic orobabilistic framework for structural health prognostic and uncertainty management. Mechanical Systems and Signal Processing, vol. 28, pp. 622-637. doi: 10.1016/j.ymssp.2011.10.019

Camci, F., Medjaher, K., Zerhouni, N., and Nectoux, P. (2013). Feature evaluation for effective bearing prognostics. Quality and Reliability Engineering International, vol. 29, pp. 477-486. doi: $10.1002 /$ qre. 1396

Hu, C., Youn, B.D., and Wang, P. (2012). Ensemble of data-driven prognostic algorithms for robust prediction of remaining useful life. Reliability Engineering and System Safety, vol. 103, pp. 120-135. doi: 10.1016/j.ress.2012.03.2008

Medjaher, K., Camci, F., and Zerhouni, N. (2012). Feature extraction and evaluation for health assessment and failure prognostics. First European Conference of the Prognostics and Health Management Society, Dresden, Germany, July 3-5, 2012.

Sawalhi, N., and Randall, R. (2004). The application of spectral kurtosis to bearing diagnostics. Proceedings of ACOUSTICS, November 3-5, Gold Coast, Australia, pp. 393-398.

Antoni, J. (2006). The spectral kurtosis: A useful tool for characterizing non-stationary signals. Mechanical Systems and Signal Processing, vol. 20, pp. 282-520. doi:10.1016/j.ymssp.2004.09.001

Nikolaou, N.G., and Antoniadis, I.A. (2002). Demodulation of vibration signals generated by defects in rolling element bearings using complex shifted Morlet wavelets. Mechanical Systems and Signal Processing, vol. 16, pp. 677-694. doi:10.1006/mssp.2001.1459 
Wang, Y., and Liang, M. (2011). An adaptive SK technique and its application for fault detection of rolling element bearings. Mechanical Systems and Signal Processing, vol. $25, \quad$ pp. 1750-1764. doi:10.1016/j.ymssp.2010.12.008

Howard, I. (1997). Higher order spectral techniques for machine vibration condition monitoring. Proc. of IMechE - Part G, vol. 211, pp. 211-219. doi: $10.1243 / 0954410971532622$

Jiang, L., Liu, Y., Li, X., and Tang, S. (2011). Using bispectral distribution as a feature for rotating machinery fault diagnosis. Measurement, vol. 44, pp. 1284-1292. doi: 10.1016/j.measurement.2011.03.024

Gelman, L., White, P., and Hammond, J. (2005). Fatigue crack diagnostics: A comparison of the use of the complex bicoherence and its magnitude. Mechanical Systems and Signal Processing, vol. 19, pp. 913-918. doi: 10.1016/j.ymssp.2004.07.009

Li, C., Ma, J., and Hwang, B. (1996). Bearing condition monitoring by pattern recognition based on bicoherence analysis of vibrations. Proc. of IMechE - Part C, vol. 210, pp. 277-285. doi: 10.1243/PIME_PROC_1996_210_197_02

Yang, D., Stronach, A., and MacConnell, P. (2002). Third order spectral techniques for the diagnosis of motor bearing condition using artificial neural networks. Mechanical Systems and Signal Processing, vol. 16, pp. 391-411. doi: 10.1006/mssp.2001.1469

Webb A. (2003). Statistical Pattern Recognition, Johan Wiley \& Sons Ltd.

Gelman, L., Lapena, E., and Thompson, C. (2009). Advanced higher order spectra for classification of damage in transient conditions. Journal of Intelligent Material Systems and Structures, vol. 20, pp. 13431349. doi: 10.1177/1045389X08097383

Collis, W., White, P., and Hammond, J. (1998). Higher order spectra: Bispectrum and Trispectrum. Mechanical Systems and Signal Processing, vol. 12, pp. 375-394. doi: $10.1006 / \mathrm{mssp} .1997 .0145$ 